\title{
Technological Deconstruction and Reorganization Process of Industrial Design Driven Product Innovation
}

\author{
Wu Qiong ${ }^{1}$, Yin You-tao ${ }^{2 *}$ \\ ${ }^{1}$ Art Design College, Nanjing Tech University, Nanjing, Jiangsu, 211816, China \\ ${ }^{2}$ Art Design College, Nanjing Tech University, Nanjing, Jiangsu, 211816, China
}

\begin{abstract}
The work aims at discussing the essence of technology deconstruction and reorganization, and establishing the core value of technical decision-making in product micro-system design, In view of the current reality of neglecting product technology innovation. It clarifies the content of product technology and explores the ways and methods of product technology deconstruction and restructuring in the industrial design process. Industrial design is integration and inclusion, parallel design and collaborative design is the overall trend of its development, product technology design and modeling, man-machine and other design will eventually become unified. The innovation of working principle is the process of replacing the basic elements and modules or optimizing the state of material, information and energy transfer; product structure optimization is the process of mathematically finding the minimum design variables that meet the design requirements; the innovation of modeling material solutions is to solve the decision-making problem of the target multi-scheme; the processing technology improvement is to solve the process route optimization problem under multi-constraint conditions.
\end{abstract}

\section{Introduction}

Industrial design is based on user needs, technical analysis of product functions, structure, materials and processes, fusion of humanities and art concepts, and system integration innovation to achieve comprehensive design innovation of products[1]. Technology is the key element that constitutes a product, it is the necessary means to realize the practical function of the product, and it is the core of product innovation. The product's technical innovation design often adopts the three-step method of decomposition, technological improvement, and restructuring, which is called the "technical composition" method. According to the norms of plastic arts, the technical decomposition is called "technical deconstruction", and the technical improvement and reconstruction is called "technical reorganization"[2]. The deconstruction and reorganization of product technology is the necessary process for industrial design to assist engineering technological innovation and achieve design innovation.

\section{Product technology composition}

Technology is the key to product information transmission, including basic principles such as product working principle, technical route, choice of modeling materials and design of processing technology. The working principle is the method and method adopted by the product in order to achieve its practical functions; the technical route is the structural system composed of product parts according to the working principle; the modeling material is the material basis for the product component molding; the processing technology is the processing of materials into components Production technology.

\section{Approaches and methods of product technology deconstruction}

\subsection{Practical function research}

Product practical function investigation is a process in which industrial designers put aside the spiritual function and analyze the practical function of the material level. Practical function research needs to meet the following requirements: clear research content (main functions, auxiliary functions, identification, use, and ease of operation of the product); diversified research methods, in addition to traditional questionnaire methods, experimental methods, observation methods, etc In addition to the basic method, you can use the advantages of data analysis in the era of big data to capture functional information that is not easy to find by the human eye[3]; statistically evaluate the functional evaluation results scientifically, taking into account user experience, online reviews, and feature selling points in advertising, etc. The analysis report must be objective, truthful and comprehensive. 


\subsection{Analysis of working principle}

A comprehensive understanding of the working principles, functions, and usage status of the product is the first task in product design.

(1) Product manual. In addition to the detailed description of the product's practical functions, operation and maintenance methods, the product manual will also briefly introduce the working principle of the product.

(2) Physical anatomy. Physical anatomy is the most direct and accurate way to understand how a product works. Dissecting the product does not mean destroying it. It must be carried out in order from the outside to the inside, from simple to complex.

(3) Document Retrieval. Designers can refer to relevant information to reduce barriers to knowledge in order to better master the principle of the product. This process is technical innovation, providing accurate and reliable information for principle analysis and design.

(4) Expert interview. Consulting experts can help industrial designers make up for shortcomings in professional knowledge. At the same time, the opinions of experts have important reference values in the later working principle design.

(5) Deductive reasoning. When the anatomical feasibility of the existing product is low, the working principle of the existing product can be indirectly estimated by referring to similar or similar products.

\subsection{Product Structure Anatomy}

Industrial products have extensive extensions and rich connotations, and are generally composed of three parts: function, form and structure. Each of the three parts has its own irreplaceable content, which restricts and cooperates with each other. The anatomy of a product is a process from awareness to perception, an iterative process between rationality and perception[4].

\subsubsection{Understanding the product structure}

Disassembly and mapping of small and medium-sized products. For small and medium-sized products, industrial designers must disassemble the product and map the relevant parts in a targeted manner, as shown in Figure 1;require an understanding of the main

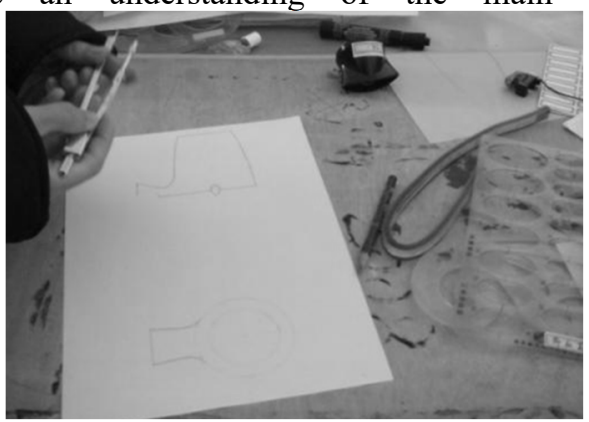

dimensions of each component (the main dimensions involved here are: length, width, height, maximum size, minimum size, inclination, radius and corresponding allowable error range of the component); have a rational understanding of the mutual combination between internal structures.

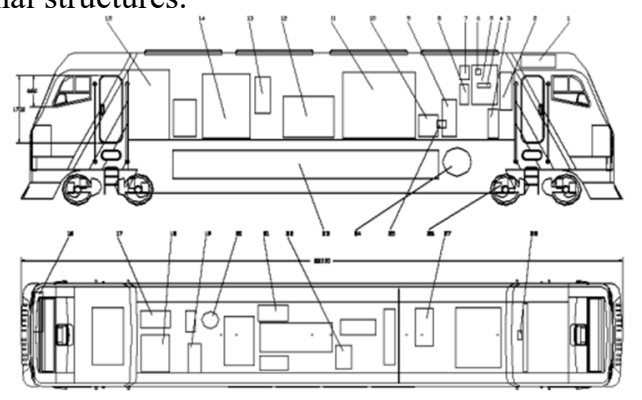

Fig 1. Product disassembly and mapping

Large-scale product assembly and illustration. It is impossible for industrial designers to disassemble and survey large-scale products separately. They can make full use of the company's existing drawings and corresponding documents to the assembly production line to directly understand the size and combination of each component of the product.

\subsubsection{Perceiving the product structure}

Product design assembly drawing. Dismantling surveying and on-site illustration only have a more perceptual understanding of the product structure based on the working principle. To truly understand the internal mechanism of the product structure, it is necessary to go through the process of product design assembly drawing. The design drawing of the industrial designer mainly achieves three purposes: the size of the key parts of the product modeling and other parts; the logical relationship of the combination of the product parts; the maximum tolerance size of the internal components of the product.

Black box processing of complex functional parts. Use the principle of maximum containment size to draw the black box, as shown in Figure 2. The size of the black box is inversely proportional to the depth of technical understanding. Theoretically, the smaller the black box, the greater the space for future shape design.

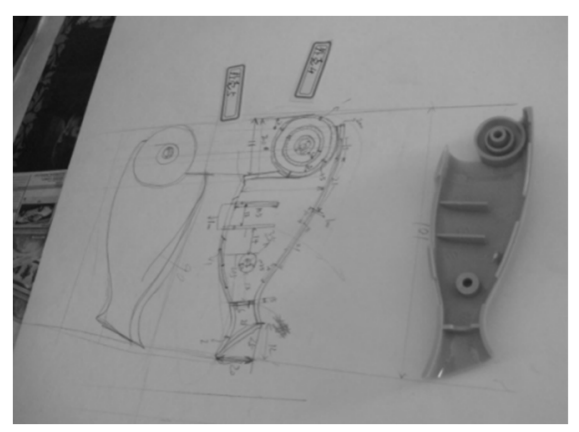

Fig 2. Black box processing 


\subsection{Analysis of modeling materials}

Industrial design is the organic unification of technology and art, and has both physical and spiritual characteristics. Therefore, the choice of modeling materials for industrial products must have the unique characteristics of modeling materials in addition to the general material properties required by the product's practical functions.

(1) Sensory characteristics. The comfort, intimacy, and warmth of materials have a special expressive force on the surface decoration of industrial products, and are the elements that cannot be ignored in the beauty of product modeling.

(2) The culture of the times. Modern design pays more and more attention to the cultural value and emotional appeal of products to users. The cultural nature of materials is that the materials are precipitated in long-term use and conform to the values of the spirit of the times. For example, wood usually conveys natural and elegant semantics, and metal conveys modern, rational semantics under industrial civilization.

\subsection{Processing technology analysis}

Processing technology is the core technology of product molding. Production technology refers to the techniques and methods that people use in production labor to process, process (such as forging, cutting, heat treatment, etc.) and assemble labor objects (various raw materials, reproducts), and finally become products. The investigation of production process means to fully understand the existing production process, in order to make full use of the characteristics of the production process in the next design.

(1) Field trip. A field trip is a straightforward and effective method. The content of the inspection includes the forming process, surface treatment process and assembly process of parts, and even the packaging and transportation process of products.

(2) Engineering personnel communication. Understand the corresponding production process of parts through communicating with experts and engineers.

(3) Borrow from reasoning. For new products (concept products), some processes are difficult to find in the production process of similar products, and it can be deduced from other products with similar functions.

(4) Process innovation. Through searching journals, consulting experts, etc. to help understand the latest developments in process development and optimize production processes in a timely manner.

\section{Ways and methods of product technology reorganization}

\subsection{Function and working principle innovation}

\subsubsection{User requirements and functional design}

User experience is the starting point and ending point of functional design. Through the "designer-user-product" interaction, a user-participative design mode is established, which analyzes the problems, needs and suggestions of users when using the product, and determines the goals of functional design and optimization vision[5].

\subsubsection{Principle decomposition and dynamic relationship analysis}

Determine the corresponding working principle system model around the function and draw the functional principle diagram. The functional system is decomposed by the principle of system engineering decomposability according to the total function, sub-function, and functional element, and the principle modules are divided, and the function tree diagram is decomposed[6]. Functional principle decomposition can help designers distinguish the principle system components so as to determine the area to be optimized. The functional schematic diagram describes the actions and processes of product function realization; the functional tree diagram describes the composition and hierarchical relationship of functional systems.

The working principle is a process system, in which the subsystems and elements are arranged in an orderly manner and are interconnected and restricted to ensure that the functionality of the product can be correctly implemented. Industrial designers need to clarify the symbiosis and containment relationship between the internal elements of the product functional system, establish a functional relationship information catalog, and provide optimized nodes and areas for functional elements or modules. For example, the replacement of energy modules on vehicles has resulted in a variety of new energy vehicles, as shown in Figure 3. The form and state of material, energy and information conversion between systems are also important analysis and optimization directions, and product disruption and innovation often come from this. For example, the innovation of the working principle of the french fries machine is a change from cutting to pressing and injection molding into french fries, which has changed the state of material transmission, and has produced subversive innovative products. 



Fig 3. Replacement of energy modules in the working principle of vehicles

\subsubsection{System reconfiguration and optimization}

By introducing or replacing new functional elements, the organic combination of replaceable functional element solutions in the principle system is found, the structure and content are optimized, and the multi-solution scheme group of the working principle system is established. According to the basic principles of specific products, design constraints and technical contradictions in the process of restructuring the principle system, a mathematical model is established, and "TRIZ" and other theories are used appropriately to test and analyze the functional principle scheme group. Finally, the working principle is derived based on engineering practice Optimal solution for system optimization design.

The development of products generally follows the development route of rigid, hinged, flexible, fluid, and field. For example, the development of the mouse has experienced a roller ball mouse, to an optical mouse, to a laser mouse, and to a field-driven wireless mouse and a Bluetooth mouse. Designers can refer to the evolutionary principles of the eight major technology systems during the reconstruction of working principles to determine the evolutionary stages of the working principles of current product groups, explore existing or potential problems with design objects at this stage, and make innovative predictions of principles, using as few resources as possible. Achieve more optimized functional goals.

\subsection{Product structure optimization}

The main purpose of product structure optimization is to improve structural performance and increase safe life on the premise of ensuring product performance and cost control. The development of computer simulation technology has promoted the visualization and parameterization of the structural design process, optimized the quality of the design, and greatly shortened the product development cycle. The finite element analysis method regards the solution domain as composed of many interconnected finite element domains, assumes an appropriate approximate solution for the element, and then derives the total satisfaction conditions of the solution domain to obtain the solution of the problem[7]. The 3D computer simulation technology based on the finite element analysis method is the main method for optimizing the product structure, as shown in Figure 4. 




Fig 4. Structural optimization process of 3D computer simulation technology based on finite element analysis

\subsubsection{Structural prototype design and model creation}

The designer prototypes the layout and type of the structure based on professional knowledge, experience and material characteristics, and builds models through parametric modeling software such as CAITIA and CAD.

\subsubsection{Finite element analysis and function solving}

FEM analysis software such as ANSYS and MARC are imported through the seamless interface for related processing. The main processing processes include finite element meshing, environment definition, material definition, boundary definition, etc.

Look for quantities that reflect design requirements, such as uniform stress distribution, lightening, etc. Design variables in structural optimization are generally physical and geometric parameters, such as wall thickness and pillar position in the product will greatly affect the structural performance of the product, and even cause certain harm to the product performance. The objective function is designed according to the discriminant index, the structure limiting variables are analyzed, and the constraint conditions are determined. Find the discrete relationship of design variables, continuously deriving design variables, introduce finite element analysis software to modify the threedimensional model of the product, seek the best solution among the obtained structural parameters and response results, and obtain a reasonable product structure optimization plan.

\subsection{Innovation in modeling materials}

The combination of modeling materials is diversified due to the multi-directionality and uncertainty of the selection criteria in product design. Facing the decisionmaking problem of modeling materials as multi-objective and multi-plan, designers usually need to select the best combination of solutions based on the material semantics of the current era and combine the analytic hierarchy process, and establish membership matrix and evaluation according to specific design needs and constraints. The matrix provides a more scientific reference for the innovation and design of product modeling materials. Analytic hierarchy process is a multi-objective decision analysis method that combines qualitative and quantitative analysis methods[8]. The main idea of this 
method is to decompose complex problem levels and factor levels, compare the importance of a pair of criteria indicators layer by layer, establish a corresponding judgment matrix, and calculate the importance ranking between different solutions according to the calculation, which is the best solution. Choose to provide a basis.

\subsubsection{Building user and material semantic directories}

The designer builds a material semantic catalog based on the relevant materials involved in a specific design. The material conveys its practical and cultural information to the user. Such as stainless steel pots, the physical and chemical properties of the material itself meet the requirements as a food tool, and at the same time convey to the user a refined, pure cultural semantics[9]. The designer needs to investigate the operating habits and methods of the target user and establish a user semantic model. The user semantic model is mainly the user's cognition and operation of materials in the current social stage, and the subjectivity and variability are mostly; the material semantic model is the objective value analysis of materials by professional designers.

\subsubsection{Building a Hierarchical Model and a Hierarchy Judgment Matrix}

The hierarchy model mainly includes the target layer, the criterion layer and the scheme layer, as shown in Figure 5. The target layer corresponds to the ideal solution for the modeling material. The criterion layer is usually the design criteria such as craftsmanship, physicochemical, cultural, economic, and environmental protection. A prototype of a modeling material program designed with a semantic catalog of materials.

A comparison between pair of design criteria is adopted for the target layer and the scheme layer to construct a judgment matrix. The scale definition of the evaluation criteria in the judgment matrix needs to be formulated with the collaborative participation of designers, engineering and technical personnel, enterprise management personnel, and users. At the same time, a reasonable interval definition needs to be based on specific design projects.

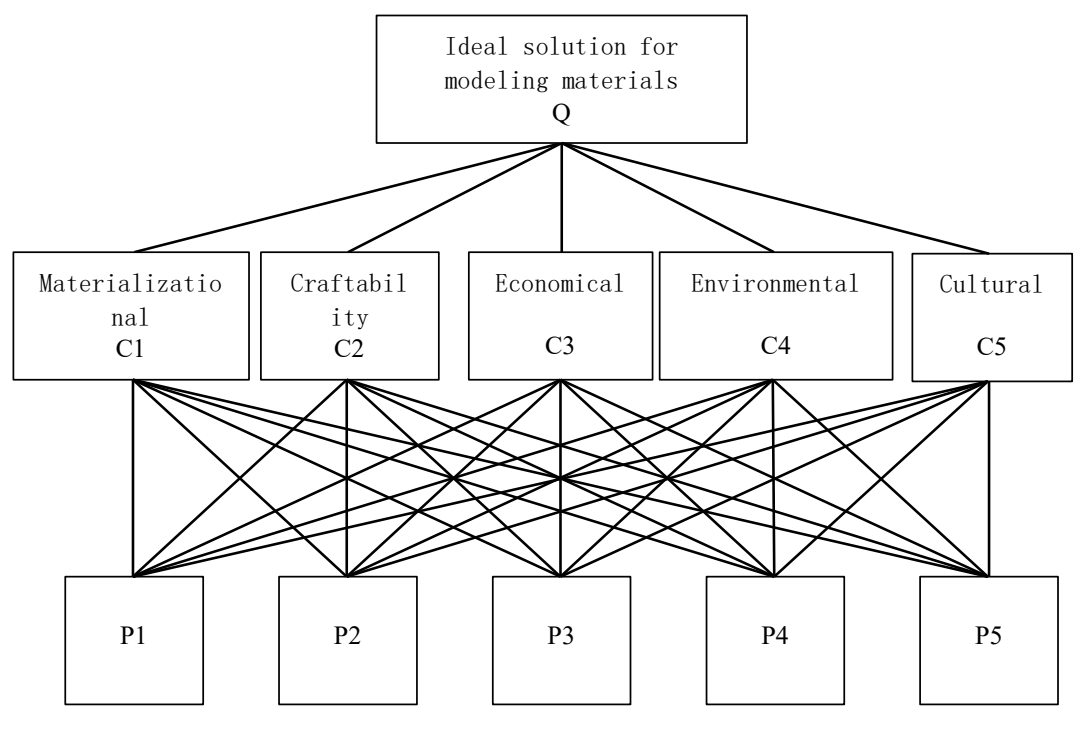

Fig 5. Modeling structure diagram of modeling materials

\subsubsection{Sorting and consistency check}

The approximate and maximum eigenvalues of the matrix eigenvalues are calculated, and the index weights of each layer are listed. The size of the index weights expresses the weight relationship between the design criteria in each material prototype scheme. The degree of matrix error is judged by checking the consistency of the matrix. On the premise of satisfying the consistency, the index weights between each material scheme are obtained, and the order of the advantages and disadvantages of the modeling material scheme combination is determined.

\subsubsection{Verification and evaluation}

The definition scale of the evaluation criteria for modeling materials is a qualitative process in this process. At the same time, this method can only evaluate and screen the prototype of the material combination, so it cannot provide a new design solution. Therefore, the professional literacy requirements of the relevant participants are relatively high. The optimal solution selected still needs to be verified with the participation of users and corporate personnel.

\subsection{Process improvement}

The processing technology of a product is linked to the design and manufacture of the product, and is a means of 
value-added design information. The material and structural characteristics of product parts determine the choice of product processing method and manufacturing resources. At this stage, the design of machining process is more mature than that of plastic. It is beneficial to shorten the construction period and improve the yield rate.

\subsubsection{Verification and evaluation}

Product processing features are the carrier of product component processing information. They can be divided into two types: main features and auxiliary features. The main feature is the geometric topological information of product parts. The auxiliary features are local features attached to the main features[10]. The processing information of product parts mainly includes parts characteristics, processing stages, processing methods, processing resources, and fixture locations. The processing element is the smallest unit of product processing. Establishing a product component processing meta-catalog facilitates the representation of product characteristic information and processing information.

\subsubsection{Process definition and optimal solution}

Define the product process according to the parts processing meta-catalog, draw the parts process route model, and convert the optimization problem of the process route into different constraints in the process order according to the processing requirements, such as processing method selection, machine tool selection, tool selection, fixture selection, process Under the constraints such as constraints. it seeks the processing metaoptimization ordering problem that makes the target the best[11]. According to the specific product processing technology, processing properties and the design constraints, the designer needs to choose the corresponding sorting method.

\section{Conclusion}

Industrial design is a systematic project to solve the problem of users' value demand. The deconstruction and reorganization of the system provide a "trade-off" design philosophy, which drives the deconstruction of the technical system and the reorganization of the minimum information elements. It is the core value of industrial design-driven technology decision-making that how to make quantitative judgment under multiple constraints in complex scenarios. Design is always changing, designers should find the dynamic combination of constraints in the product microsystem, explore the maximum boundary of product value, and push the "mechanism" of manufacturing transformation and upgrading.

\section{Acknowledgments}

Authors are very grateful for the support of the design methodology provided by Nanjing Lincy Industrial
Design Co., Ltd. and the financial support provided by the Jiangsu Social Science Foundation key project "Design Semiology" (18YSA001).

\section{References}

1. WU Qiong.Quality Control of Large-scale Electromechanical Product Model Design[J]. Mechanical Design,2014(8):115-118.

2. WU Qiong.Thinking based on the specific connotation and operation mode of enterprise product innovation design[J].Enterprise Economy,2009,6:31-33.

3. Ren Yingli, Fan Qiang.Applicability of Big Data in Product Design and Research[J]. PackagingEngineering, 2015, 36(10):139-142.

4. CHU, CHIH-H, LU Yuanping,et al.Economical green product design based on simplified computeraided product structure variation[J].Computersin Industry, 2009, 9(60):485-500.

5. TAN Hao,LI Wei,HAN Lihong.Study on Userinvolved Industrial Design Generation Thinking[J].Packaging Engineering,2018,39(24):146-151.

6. ZHANG Peng, DONG Yafan, ZHANG Gaogao, et al. The design process complexity theory and TRIZ theory integrated complex electromechanical system functional decomposition process model[J]. Journal of Mechanical Engineering, 2016, 52(23): 17-24.

7. WU Xiaojun, HU Guilin, ZHOU Chunhao, et al. Structural Design and Optimization of Insulation Box for Frozen Seafood Products[J]. Packaging Engineering, 2018, 39(3): 72-76.

8. ZOU Tao,SHI Wei.Application of Analytic Hierarchy Process in Children's Bicycle Design[J].Packaging Engineering,2019,40(02):161166.

9. LIU Shengzhi, Zhu Zhongyan. Product Semantics and Product Design[J].Packaging Engineering,2006(01):182-184+195.

10. LI Congbo, LI Pengyu, LIU Fei, et al.Multiobjective optimization model for high-efficiency and low-carbon machining process route[J].Journal of Mechanical Engineering,2014,50(17):133-141.

11. CHANG Zhiyong, YANG Jianxin, ZHAO Jie, et al. Process Optimization Based on Adaptive Ant Colony Algorithm[J]. Journal of Mechanical Engineering, 2012, 48(9): 163-169. 